\title{
A Meta-Analysis: The Effects of CBT as a Clinic- \& School-Based Treatment on Students' Mathematics Anxiety
}

\author{
Ali Bicer ${ }^{1^{*}}$, Celal Perihan ${ }^{2}$, Yujin Lee ${ }^{3}$ \\ ${ }^{1}$ University of Wyoming, USA \\ 2 Idaho State University, USA \\ ${ }^{3}$ Indiana University-Purdue University, USA \\ *CORRESPONDENCE: - abicer@uwyo.edu
}

\begin{abstract}
The purpose of this meta-analysis was to examine the effects of Cognitive Behavioral Therapy (CBT) interventions as a clinic-based treatment on students' mathematics anxiety level. A total of 7 studies containing 14 effect sizes were selected for inclusion in the present meta-analysis. Results indicated that the overall effect size was moderate to strong $(g=-0.76$ with $S D=0.04)$. The implication from the present study is that CBT can be a useful intervention to reduce students' mathematics anxiety.
\end{abstract}

Keywords: mathematics anxiety, mathematical fear, cognitive behavioral therapy

\section{INTRODUCTION}

Mathematics anxiety can be defined as the emotional reaction of fear, tension, helplessness, and mental disorganization when dealing with a mathematics problem (Ashcraft, 2002; Fennema \& Sherman, 1976; Zettle, 2003). More generally, this negative emotional reaction or feeling may also be experienced toward mathematics itself. In other words, mathematics anxiety can be considered as a negative feeling toward mathematics or the prospect of doing mathematics (Anderson, 2007; Hembree, 1990; Lyons \& Beilock, 2010). Mathematics anxiety among students has been a global concern as international comparisons of students such as the Trends in International Mathematics and Science Study (TIMSS) indicated that students in many countries experienced mathematics anxiety (Beilock \& Willingham, 2014).

Mathematics anxiety can have a substantial physical, psychological, and educational impact on students. Students who had mathematics anxiety showed diverse symptoms such as nausea, stomachache, and concentration issues (Kitchens, 1995; Uusimaki et al., 2004). Furthermore, when experienced long-term, mathematical anxiety negatively impacted students' mathematics achievement as well as their academic success in college (Erden \& Akgul, 2010; Foss \& Hadfield, 1993; Vitasari, Herawan, Wahab, Othman, \& Sinnadurai, 2010). Given the long-term damaging effects of mathematics anxiety, determining what interventions are effective in reducing mathematics anxiety is essential.

Teachers can adopt one of several research-based interventions in their classrooms that may help lessen their students' mathematical anxiety, thereby increasing students' positive attitudes toward mathematics. These interventions include but are not limited to cooperative and small grouping (Duffy \& Furner, 2002; Lee, Capraro, \& Bicer, 2019), use of manipulatives and technology-based instruction (Bicer et al., 2018), the identification of problem areas and use of proactive positive approaches (Fotoples, 2000), and incorporation of journal writing and bibliotherapy (Duffy \& Furner, 2002). These interventions have reduced students'

Article History: Received 30 November $2019 \bullet$ Revised 3 January $2020 \bullet$ Accepted 3 January 2020

(C) 2020 by the authors; licensee Modestum Ltd., UK. Open Access terms of the Creative Commons Attribution 4.0 International License (http://creativecommons.org/licenses/by/4.0/) apply. The license permits unrestricted use, distribution, and reproduction in any medium, on the condition that users give exact credit to the original author(s) and the source, provide a link to the Creative Commons license, and indicate if they made any changes. 


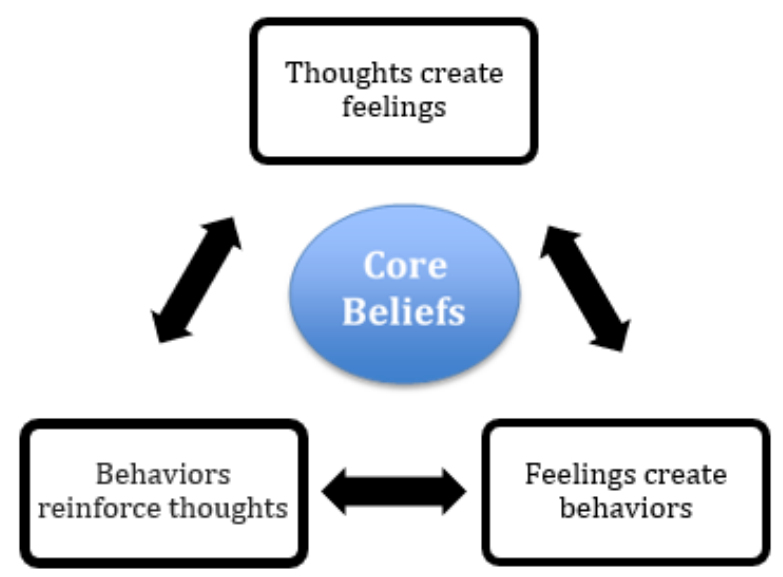

Figure 1. Cognitive Behavioral Therapy

mathematical anxiety, and eventually improved their mathematics achievement (Beilock \& Maloney, 2015; Hembree, 1990; Ramirez, Chang, Maloney, Levine, \& Beilock, 2016; Zakaria, Zain, Ahmad, \& Erlina, 2012). One of the most effective interventions focused on reducing the level of anxiety in children is CognitiveBehavioral Therapy (CBT).

CBT is an evidence-based practice for internalizing a variety of problems in children with or without disabilities (Kendall et al., 2005; Manassis et al., 2010). The core CBT was developed by Beck in 1976 to treat anxiety and depression symptoms, and has been used to treat other types of problems or disorders such as anger, stress management, and bipolar disorders (Hofmann Asnaani, Vonk, Sawyer, \& Fang, 2012). The main goal of CBT is to teach individuals how to replace their automatic thoughts with more realistic and meaningful thoughts to change core beliefs that affects an individual's thoughts and behaviors (Beck, Davis, \& Freeman, 2015). Thus, during the CBT sessions, therapists work with clients (1) to identify the thoughts and beliefs that cause anxiety with psychoeducation sessions, (2) to analyze the relationship between thoughts and anxiety with cognitive-based interventions, and (3) to teach coping techniques to control and minimize the problem behaviors (Barrett, Dadds, \& Rapee, 1996; Kendall et al. 2005). The CBT can be delivered with individual or group formats with a variety of lengths, up to six months (James, James, Cowdrey, Soler, \& Choke, 2013). In some cases, intervention can extend beyond six months and include the application of "booster sessions" to help children manage, generalize, and internal problems in new situations (Seligman \& Ollendick, 2011).

Numerous studies have demonstrated the efficacy of CBTs on different types of problems across different settings (e.g., Perihan et al., 2019; Schneider, 1993; Wadlington, Austin, \& Bitner, 1992; Zettle, 2003). To date more than 250 meta-analyses were conducted to demonstrate the effects of CBT for a variety of disorders including anxiety-related disorders, and they reported that CBT had significant beneficial effects for children in clinical settings. Although a number of studies have demonstrated the effects of CBT in a variety of problems in children and adults in clinic settings, recent studies have transferred CBT from clinical to school settings with modifications to treat children in school settings, and moderate to large effects have been obtained for controlling anxiety symptoms with school-based CBT compared to control groups (Bernstein, Layne, Egan \& Tennison, 2005; Neil \& Christensen, 2009). Within this emerging evidence, CBT has also been one of the potential interventions for lessening mathematical anxiety (Hembree, 1990; Karimi \& Venkatesan, 2009; Lyons \& Beilock, 2010; Maloney, 2012; Zettle, 2003). Underlying CBT is the idea that thoughts create feelings, feeling create behaviors, and behaviors create thoughts (see Figure 1). Therefore, applying CBT as an educational intervention in mathematics classes may have a substantial and direct impact on students' mathematics anxiety because students' mathematical thoughts, feelings, and behaviors are interrelated.

However, there has been no published meta-analysis showing the effects of CBT in reducing students' mathematics anxiety. The purpose of the present study was to investigate the effects of CBT intervention on students' mathematics anxiety by conducting a meta-analysis. 


\section{THEORETICAL FRAMEWORK}

\section{Cognitive Behavior Therapy (CBT) Interventions on Mathematics}

CBT was developed by Aaron T. Beck in the 1960's and is defined as an outcome-focused, client-centered, collaborative treatment designed to address individuals' problems, emotions, thoughts, and behaviors (Beck, 1976). CBT programs typically include using cognitive interventions to change automatic beliefs, and behavioral intervention for coping with the anxiety symptoms (Kendall \& Hedtke, 2006). The primary purpose of CBT is to support individuals by teaching them cognitive and behavioral strategies that will foster and improve their well-being (Cayoun, 2014; Kendall, 1993). As of today, several types of child and adolescent CBT programs have been used in school settings, such the 'Coping Koala' (Barrett et al.,1996), 'Skills for Academic and Social Success' (Warner, Warner, Fisher, \& Klein, 2007) and many of modular format CBTs. Many researchers have investigated the effects of various pedagogical interventions on reducing students' mathematics and test anxiety, but few have examined how students' cognition and behavior change in relation to their mathematics anxiety after receiving CBT intervention. One reason for disregarding the effects of CBT on reducing students' mathematics anxiety might be that "most researchers, however, consider mathematics anxiety to be a construct that is distinct from attitude towards mathematics" (Ma, 1999, p. 520). On the other hand, studies related to mathematics anxiety noted that mathematics anxiety has two forms: affective (e.g., attitudinal and emotional element) and cognitive (Hart, 1989; Ho et al., 2000; Wigfield \& Meece, 1988; Wei, 2010; Zettle, 2003). CBT as an intervention involving both affective and cognitive elements of students' learning should be considered to cope with students' mathematics anxiety.

\section{CBT \& Mathematics Anxiety}

Mathematics anxiety has an affective dimension and a cognitive dimension. Affective mathematics anxiety is an emotional component of anxiety that refers to nervousness, tension, fear, and unpleasant physiological reactions toward mathematics (Uusimaki et al., 2004). On the other hand, cognitive mathematics anxiety pertains to an individual's negative expectations and self-deprecatory thoughts toward an anxiety-causing situation in mathematics (Ho et al., 2000; Sarason, 1987; Vukovic, Kieffer, Bailey, \& Harari, 2013). Findings from previous studies have indicated that affective mathematics anxiety and cognitive mathematics anxiety had a more or less significant relationship to students' mathematics performance in both positive and negative directions (Deffenbacher, 1978; Ho et al., 2000; Sharma \& Rao, 1983; Vukovic et al., 2013). Although affective mathematics anxiety has been primarily shown to have a negative correlation with students' mathematical performance, cognitive mathematics anxiety has been mainly shown to have a positive correlation with the mathematical performance. For instance, cognitive mathematics anxiety may manifest itself in a students' concern about low achievement in mathematics. In response to this anxiety, a student may meticulously and thoroughly study, and this effort would likely result in higher academic achievement (Ashcraft, 2002; Ma \& Xu, 2004; Namkung, Peng, \& Lin, 2019; Wigfield \& Meece, 1988). However, cognitive mathematics anxiety may not always have a positive correlation with student performance; in fact, this type of anxiety could foster affective mathematics anxiety (e.g., feeling of tensions, dislike, or fear towards mathematics). A student who is worried a lot about their mathematics performance may "develop negative attitudes towards tasks involving mathematics, drop out of elective mathematics classes or avoid taking them altogether" (Devine, Fawcett, Szucs, \& Dowker, 2012, p. 2). In longer term, a student who possesses both cognitive and affective mathematics anxiety can avoid pursuing careers that require quantitative skills (Ma \& Xu, 2004). In this instance, cognitive mathematics anxiety could have a negative correlation with mathematics achievement (Ashcraft, 2002; Ma \& $\mathrm{Xu}, 2004$; Namkung et al., 2019). Therefore, researchers and educators should implement interventions focusing on reducing students' cognitive mathematics anxiety that can also reduce students' affective mathematics anxiety.

Overcoming affective mathematics anxiety through interventions targeting cognitive mathematics anxiety can enable students to achieve their potential in mathematics. One of the recent studies conducted by Birgin, Baloglu, Catioglu, and Gurbuz (2010) reported that that the highest contribution to students' mathematics anxiety was from their poor mathematics performance. Another study conducted by Ma and Xu (2004) also noted that young students who had poor mathematics performance later demonstrated high level of mathematics anxiety. All of these recent studies supported the Deficit Theory (Tobias, 1986) which claims that mathematics anxiety emerges as a result of an individuals' awareness of poor mathematics performance in the past. Being aware of prior poor mathematics performance may decrease students' self-efficacy in mathematics (Devine et al., 2012). Self-efficacy was defined by Bandura (1994) as the belief in one's ability to 


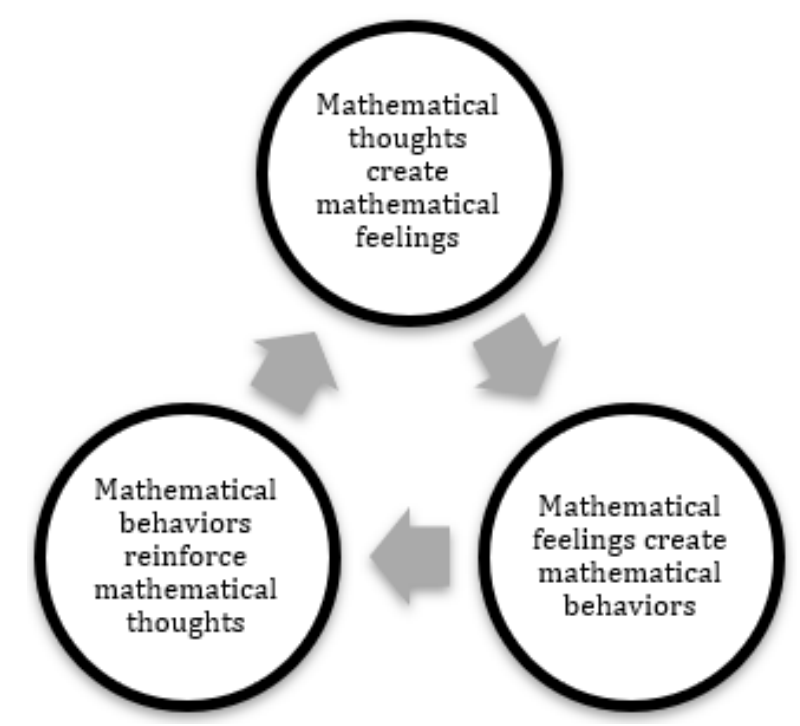

Figure 2. CBT applied to mathematics anxiety

influence course of events that can shape one's life and control over the way these events are experienced. Researchers who investigated the relationship between early mathematics achievement, mathematics anxiety, and self-efficacy in mathematics found that lower mathematics achievement could lead to lower selfefficacy in mathematics, and low self-efficacy in mathematics was one of the most vital predictors of students later increasing mathematics anxiety (Bates, Latham, \& Kim, 2011; Hoffman, 2010). Self-efficacy, as only one dimension of affect in mathematics like other dimensions (e.g, belief, value in mathematics), proved to be related to students' early performance in mathematics. The present study adopted the Deficit Theory (Tobias, 1986) claim and hypothesized that students' mathematics anxiety can be avoided by implementing an intervention (e.g., CBT) focused on reducing students' cognitive mathematics anxiety.

Cognitive mathematics anxiety and its relation to affective mathematics anxiety and mathematics performance can be explained by applying CBT in mathematics to mathematical thoughts (e.g., "I am worried about math tests because I was never successful.") that create mathematical feelings (e.g, "I dislike mathematics."), which in turn create mathematical behaviors (e.g., "I will not take any elective mathematics courses," or "I will not listen my math teacher because there is no way I can understand what he/she is describing."), and these behaviors reinforce mathematical thoughts (e.g., "I am definitely not good at mathematics and will never be successful in math."). Figure 2 demonstrates this model as an individual's mathematical thoughts refer to personal thoughts about mathematics that are interrelated with his/her mathematical feelings. Negative mathematical feelings (e.g., being worried about math test due to previous poor math performance) arise when a person performs poorly in mathematics and, based on his/her feelings, presents certain mathematical behavior (e.g., "I will not work on my math test because there is no way I can be successful."). The argument presented earlier for poor mathematics performance results from cognitive mathematics anxiety.

Mathematics anxiety can affect a student's degree of success throughout his/her education and adult life. During their education, students' mathematics anxiety hindered their ability to relate mathematics to their real-life situations; this hinderance was found to adversely impact students' mathematics aptitudes (Rossanan, 2006). In addition, students who had mathematics anxiety while completing mandatory mathematics courses were more predisposed later in their academic program to avoid mathematics-related courses offered by their school (Erden \& Akgul, 2010; Felson \& Trudeau, 1991; Vitasari et al., 2010) and abstained from pursuing professional and personal math-related activities because of their fear of performing poorly in mathematics. (Erden \& Akgul, 2010; Tobias, 1993; Vitasari et al., 2010). Decreasing mathematics anxiety could encourage students to engage in mathematics without worry and foster their interest in and pursuit of professional opportunities that involve mathematics. Therefore, students should be surrounded by a positive learning environment in which they feel completely free from tension and nervousness often associated with mathematics anxiety (Ashcraft, 2002; Miller \& Mitchell, 1994; Uusimaki et al., 2004). Fostering this positive environment will require educators to take a more proactive role in encouraging students to engage in mathematics and see themselves as successful mathematical problem solvers (Furner 
\& Berman, 2003). Hence, finding suitable and effective treatments should be a priority of educators and researchers. Without an effective treatment for reducing mathematics anxiety, students will sustain their anxiety and unreasonable beliefs (Baroody \& Costlick, 1998; Beck, Davis, \& Freeman, 2015; Kendall \& Hedtke, 2006). Therefore, an intervention focused on understanding, and if necessary, altering students' cognition in mathematics along with their behaviors and thoughts in mathematics was needed. CBT is of the educational interventions that mathematics educators adopted in an effort to reduce students' mathematics anxiety. There have been a few published studies of cognitive or behavioral interventions to deliver children with mathematics anxiety (e.g., Karimi et al., 2009; Wei, 2010). Results from these studies, in general, showed medium positive effects on reducing students' mathematics anxiety (Hembree, 1990; Karimi \& Venkatesan, 2009; Lyons \& Beilock, 2010; Maloney, 2012). For instance, Karimi et al. (2010) administered the Cognitive Behavioral Group Therapy (CBGT) to reduce mathematics anxiety in middle school students. There were 33 participants from 400 students in the range of 13-16 years old. The participants were randomly assigned either to the control group $(n=16)$ or the CBGT intervention group $(n=17)$. The CBGT group received two weekly sessions for 1.5 hours during the 15 weeks to control as well as reduce mathematics anxiety. The first three sessions were psychoeducation sessions that aimed to provide both education and information about the purpose of the program for the participants. Sessions 4 through 9 included cognitive interventions to teach students how to identify negative thoughts, and provided coping skills to manage their level of anxieties. During the last 6 sessions, the researchers taught assertiveness training and modeling methods. The preposttest was administered to demonstrate the effectiveness of CBGT with using the Mathematics Anxiety Rating Scale MARS. Results of the study showed that CBGT for reducing math anxiety is efficacious, and they found a statistically significant decrease in mathematics anxiety by post-treatment compared to the control group. Some studies also focused on specific components of CBT treatments instead of using a modular CBT. Sharp et al. (2000) used relaxation training as a coping strategy to manage the level of mathematics anxiety. The relation training was delivered the beginning of each class with 5- to 7-minute sessions across the 6 weeks. The experimental group who received treatment $(n=30)$ evidenced a significant low mathematics anxiety level at the post-treatment. Furthermore, students who received CBT demonstrated higher mathematics performance for their course subjects.

However, there have been no large scale or comprehensive meta-analytic studies published showing the general effects of CBT as a clinic-based treatment on reducing students' mathematics anxiety. Therefore, researchers in the present study aimed to examine whether CBT interventions, in general, have positive effects on reducing students' mathematics anxiety.

\section{Research Questions}

1) What are the overall effect sizes of CBT interventions on students' mathematics anxiety?

2) To what extent does students' mathematics anxiety through CBT interventions vary across the studies?

\section{METHOD}

\section{Literature Search}

The primary research question of the present study was the following: "What is the effect of CBT related intervention on reducing students' mathematics anxiety?" To address our research question, the electronic databases of ERIC, JSTOR, and CrossRef, PyschINFO, ProQest and online resources, such as Google Scholar were searched. The preferred reporting items for meta-analyses was used for the searching protocol (Moher, Liberati, Tetzlaff, \& Altman, 2009). The studies contained the following keywords: "CBT," "cognitivebehavioral therapy," "cognitive-behavioral intervention," "Mathematics Anxiety," "cognitive-behavioral therapy and mathematics anxiety", and "mathematics anxiety and cognitive-behavioral therapy". The abstract of each study was reviewed, and the studies were accepted based on the following inclusion and exclusion criteria.

\section{Inclusion and Exclusion Criteria}

Experimental and/or quasi-experimental studies that met the following inclusion criteria were included in the present meta-analysis: (1) published in English; (2) measured students' mathematics anxiety; (3) involved students in grades K-12 and college; (4) included examinations of students' fear, anxiety, and any other negative feelings toward mathematics, and (5) included at least one standardized measure of anxiety. Studies that lacked any of the inclusion criteria were excluded from the present meta-analysis. A large number of 


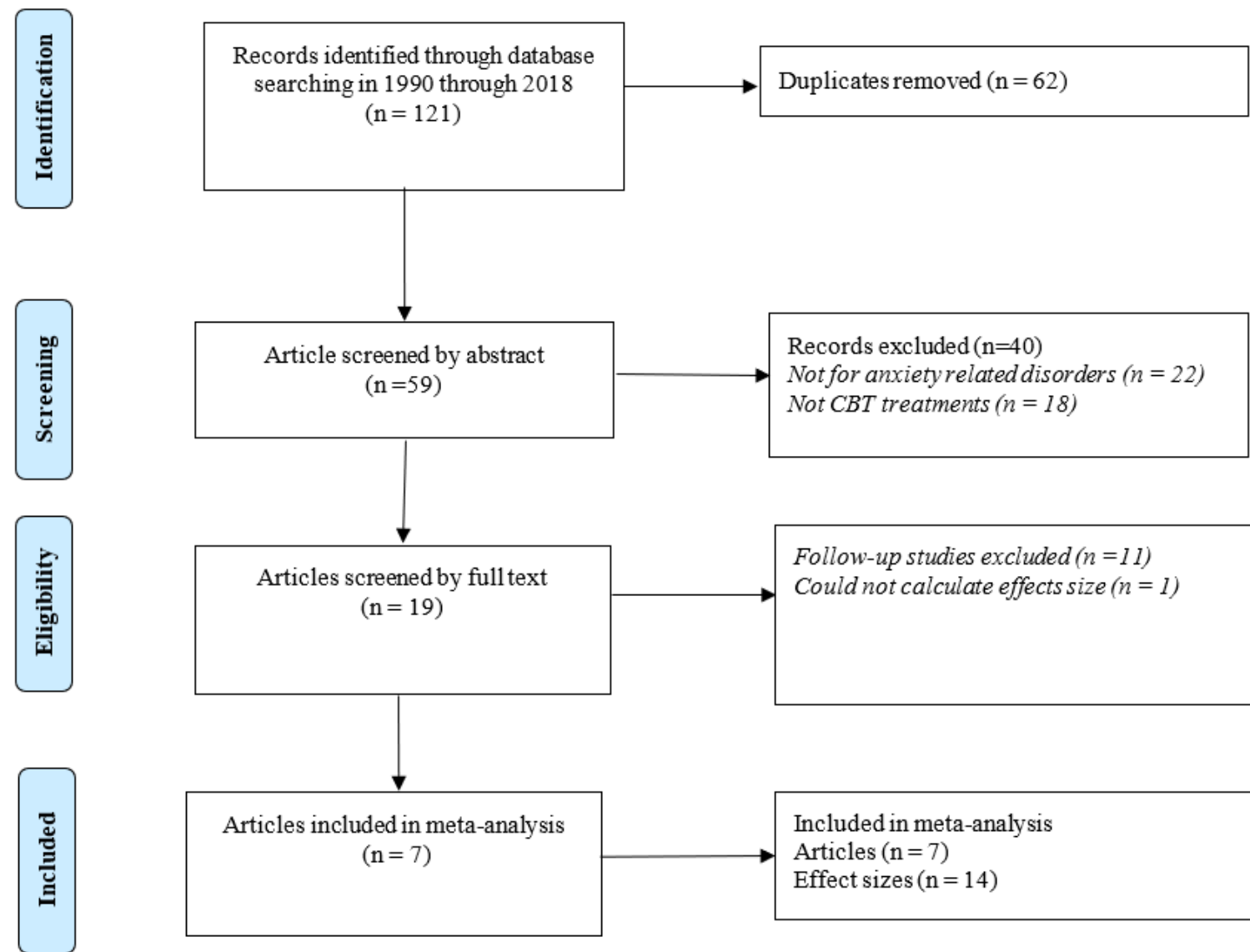

Figure 3. Selection of the studies in the meta-analysis of the effects of CBT intervention on students' mathematics anxiety

studies were excluded from this meta-analytic study because they focused on the impacts of CBT related interventions on students' general anxiety and test anxiety. Figure 3 shows a flowchart diagram for selection of the studies. The researchers also performed a backwards search using the studies cited in reviewed articles to locate additional sources. As a result, a total of 7 quantitative and mixed method studies that included 14 effect sizes that examined the impact of CBT intervention on students' mathematics anxiety were identified. Figure 3 provides an overview of the identification, screening, eligibility, and inclusion steps. To address the research quality of the experimental and quasi-experimental studies, the 27 items Downs and Black (1998) checklist was adopted. West et al. (2002) identified that Downs and Black was the best evaluation system among many other quality assessment system. The 27 items included the quality index of reporting, external quality, internal validity-bias, internal validity-confounding. Overall, according to Downs s and Black (1998)' $\mathrm{s}$ score ranges, the studies included in the present meta-analytic study received good score (20-25) on the scale of reporting (e.g. objectives, outcomes, statistical findings), external validity (generalizability), internal validity, and confounding and selection bias.

\section{Variable Coding and Coding Reliability}

Each study was coded independently for the following information; study name, sample size, participant's age, gender, intervention type, study design, treatment duration, settings, grade level, and outcome measure. House et al.'s formula (1981) was used to calculate an inter-coder agreement within coders (sum of agreement/total number of agreements + disagreements $\times 100)$. The coding procedure was done by two researchers independently and inter-coder agreement between the two coders for the variable coding was excellent, measured as $94 \%$. 


\section{Analysis of Effect Sizes}

Standard mean differences were used to compute Cohen's $d$ effect sizes from means, standard deviations, and sample sizes (Cohen, 1969). The studies involved in the present meta-analysis employed either experimental or quasi experimental design. If the studies employ true-experimental design, the effect size for each study was calculated based on students' post-test scores from surveys for mathematics anxiety. If the studies employed quasi-experimental design, the effect size for each study was calculated based on students' pre- and post-test scores from survey for mathematics anxiety (Ehrenreich-May et al., 2014; Maskey, Lowry, Rodgers, McConachie, \& Parr, 2014; Steensel \& Bogels, 2015). If there was more than one effect size within a single study, the effect sizes were averaged through robust variance estimation (RVE). Applying RVE was appropriate estimation method as the simulation studies revealed that RVE yielded accurate results with as few as 10 studies for estimating an averaged effect size (Hedges, Tipton, \& Johnson, 2010; Tanner-Smith \& Tipton, 2014). When a study reported effect sizes for different groups as multiple samples within one study, each effect size was treated as an individual study. To correct for sampling bias, all effect sizes were converted to Hedges's $g$ effect sizes, and 95\% confidence intervals were calculated (Lipsey \& Wilson, 2001).

\section{Random-Effects Model}

Random-effects models were used, as the studies' characteristics functionally differed such as the different settings, types of CBT treatments, age range, etc. Therefore, it was expected that the true effect size would be different for each study in this meta-analysis. Selecting a random-effects model was also desirable because the goal was to compute the common effect size to generalize across the high-quality studies. The randomeffects model allowed the researchers to generate results that were not found in the studies included in the current meta-analysis (Hedges \& Vevea, 1998). The Q statistics were used to identify homogeneity of effect sizes, and forest and funnel plots were used for visual demonstration, and identification of substantial outliers (Moher et al., 2009). The present meta-analysis also applied a random effects model to investigate potential moderators; however, the results of moderator analysis did not provide consistent results due to the small number of effect sizes located in the existing literature.

A total of 120 studies were identified during the researchers' electronic search. After reviewing titles and abstracts based on the inclusion and exclusion criteria and removing duplicates, 7 studies with 14 effects sizes were included in this current meta-analysis. This meta-analysis contained more effect sizes than the number of studies it included, as some of the included studies reported different effect sizes for different groups. In such studies, effect sizes of different groups were reported for the same construct; in the current study, each of these instances, when found in one of the 7 selected studies, were calculated and reported as one effect size. All studies were randomized controlled trials compared to a waitlist. These studies' sample sizes varied; however, the overall, total sample size was 2,283 . Moderator analysis was not conducted because of the small number of studies included in the meta-analysis, but a random effects model was adopted because the studies' characteristics functionally differed.

\section{RESULTS}

Results of a random-effects model on the current study showed a range in values from Hedge's $g=-2.74$ to 0.06 . Within this range, only one effect size was not negative and was very close to zero $(\mathrm{g}=0.06)$. The overall mean of the weighted effect sizes was statistically significant $\left(g=-0.76, C I_{95 \%}=-0.99,-0.53, p<.05\right)$ with significant heterogeneity, $Q(13)=83.85, p<.05$. The forest and funnel plot in Figures 4 and 5 , respectively, revealed that there is only one substantial outlier among the 14 effect sizes in the present study. However, this outlier was not excluded because the sample size of this outlier $(n=24)$ was relatively small compared to the other studies' sample sizes included in the meta-analysis, meaning that the outlier's effect would not have a substantial effect on the average weighted effect size due to small sample size of the outlier. 


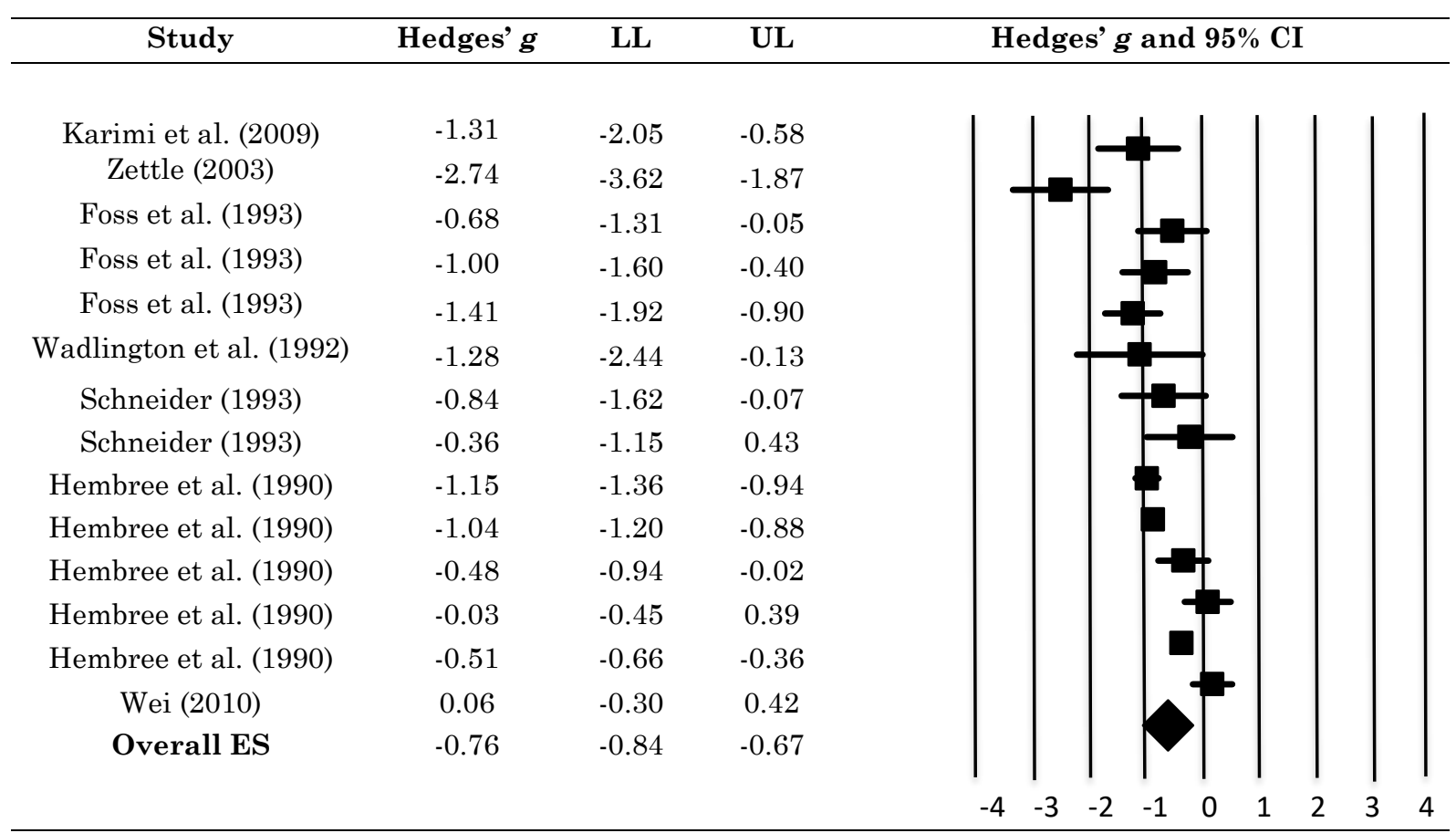

Figure 4. Forest plot of studies included in the meta-analysis. A horizontal line represents the 95\% confidence intervals of each study. The black box represents the effect size of the study. The diamond represents the overall effects across 14 effect sizes. LL = lower limit; UL = upper limit

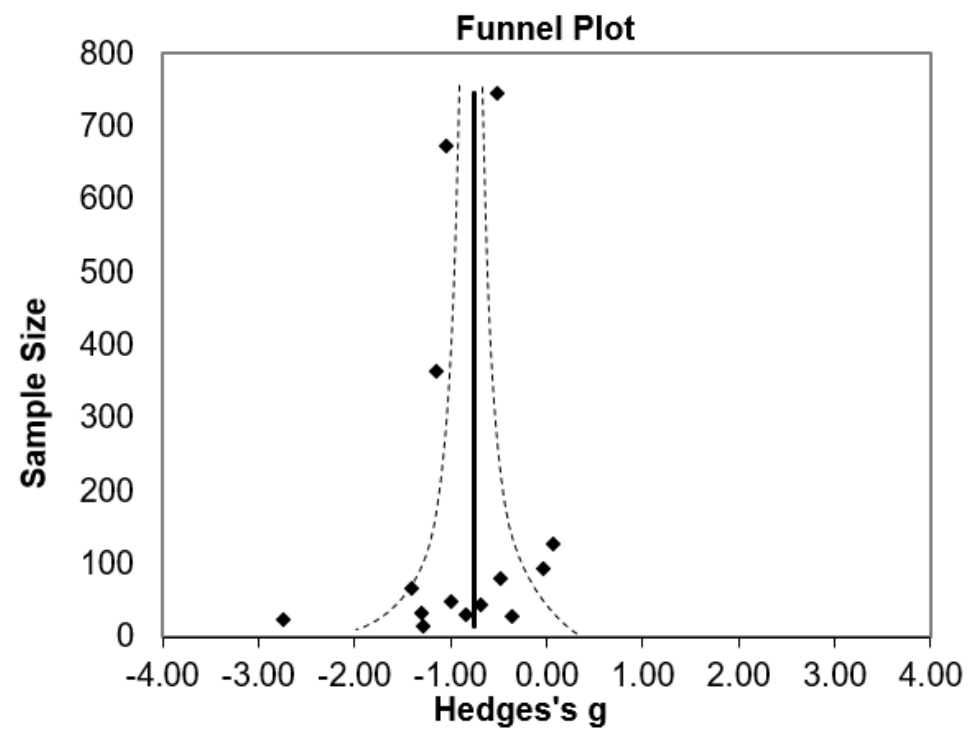

Figure 5. Funnel plot of included studies

The funnel plot (see Figure 5) also revealed that there was no publication bias in the present study. The weighted overall effect size equaled $(g)-0.64$ with $S D=0.04$. As cited in Cooper (2010) and Lipsey and Wilson (2001), Cohen identified any effect size between $(g) 0.20$ and $(g) 0.80$ as a medium effect size. Because the area of interest in the present study is the degree to which CBT intervention reduced students' mathematics anxiety, a negative mean effect size would indicate that CBT intervention was effective in reducing mathematics anxiety. Therefore, the weighted mean effect size computed here may simply reflect a medium and positive effect of CBT related interventions on reducing students' mathematics anxiety.

As stated in the literature, there have been several types of child and adolescent CBT programs, and researchers applied its various types to reduce students' general academic anxiety in school settings (Barrett et al., 1996; Warner, Warner, Fisher, \& Klein, 2007). Similarly, studies included in the present meta-analytic 
Table 1. Treatment descriptions of each study

Author (year) Treatment Descriptions

Karimi et al. Cognitive Behavior Group Therapy (CBGT) was designed to identify and cope with negative (2009) automatic thoughts. The CBGT included components of psychoeducation (3 sessions), cognitive restructuring ( 6 sessions), and behavioral activation (6 sessions). The CBGT was given in 15 sessions for 1.5 hour- two times in a week.

Zettle, (2003) Acceptance and Commitment Therapy (ACT; Hayes et al., 1999) aimed at "(a) clarifying life values and identifying barriers to realizing related goals, (b) committing to actions necessary to attain such goals, (c) evaluating private events based upon the degree to which they are useful in goal attainment, and (d) experiencing private events without engaging in counterproductive avoidance behavior (psychological acceptance)." 6 weekly sessions were administered. The First four sessions included cognitive and behavioral based treatments that aimed to create hopelessness and control the unwanted level of mathematics anxiety while the last two sessions focused to generalize the learned skills.

Foss et al. Variety of CBT components, including systematic desensitization, anxiety management (1993) training, and psychoeducation, were used to identify negative thoughts, and their effects on mathematics anxiety, and then to teach relaxation techniques to cope anxiety. The intervention also included sessions for succeeding in mathematics. The treatments were 7 to 10 weeks in duration, with the weekly session lasting one to one-half hours.

Wadlington et Systematic desensitization (SD; Wolpe, 1961), one of the behavioral therapies, was designed al. (1992) to establish a hierarchy of anxiety situations using common types of cognitive and related techniques. The SD was adapted to remove the fear response of mathematics anxiety. The intervention was demonstrated in three individual sessions lasting one-hour.

Schneider Stress inoculation (SI) training and systematic desensitization (SD) were used in the (1993) treatment of mathematics anxiety during the 6 weeks with 60 -minute sessions.

Hembree et al. Cognitive-Behavioral Treatment in this current study was a combination of cognitive (1990) restructuring with systematic desensitization to decrease the level of mathematics anxiety. The intervention included a variety of relation training and techniques to identify faulty beliefs and building self-confidence in mathematics. 60-minute individual sessions were demonstrated between 3 and 12 weeks.

Wei (2010) Cognitive-Behavioral Therapy for Generalized Anxiety Disorder (Dugas \& Robichaud's, 2007) was adopted by the author. A series of mathematics anxiety treatment messages were delivered via MathGirls, like computer software. Treatment consisted of a one-class hour for four sessions in a computer lab. The mathematics anxiety treatment messages provided detail information related to identifying of mathematics anxiety and treatment techniques.

study investigated the effects of CBT intervention on reducing students' mathematics anxiety through several CBT types (e.g., CBGT, ACT, and SD). Because the number of studies included in this meta-analysis is limited, providing a short description (See Table 1) of each CBT method applied by the studies presented in this metaanalysis is reasonable and helpful for readers to comprehend specific CBT interventions that can be used to reduce students' mathematics anxiety.

\section{DISCUSSION}

The overall effect size $(g)$ was -0.76 , and the average of the weighted mean effect sizes $(g)$ was -0.64 with $S D=0.04$. The weighted mean effect size computed here reflects a medium and positive effect of CBT related interventions on reducing students' mathematics anxiety. The finding of the present study suggests that use of CBT related interventions is helpful to reduce students' mathematics anxiety. This finding is crucial because decreasing students' mathematics anxiety could remove a barrier to pursuing employment opportunities related to mathematics and other STEM disciplines. When teachers adopt CBT related interventions to reduce mathematics anxiety, students could feel free from tension and worries often experienced with mathematics anxiety when working on mathematical problems, which may increase their desire to pursue more complex and in-depth mathematics courses (Erden \& Akgul, 2010; Miller \& Mitchell, 1994; Vitasari et al., 2010). While some level of anxiety is normal and probably useful (Wigfield \& Meece, 1988), there should be a concerted effort to identify and implement effective treatments such as CBT for reducing mathematics anxiety, because students often report paralyzing and detrimental levels of mathematics anxiety that negatively impact both their desire to pursue and engage in mathematical activities (Baroody \& Costlick, 1998; Furner \& Berman, 2003). 
The results of this study well demonstrated of the relationship between cognitive mathematics anxiety and affective mathematics anxiety, and the positive impact of reducing cognitive mathematics engagement on reducing students' affective mathematics anxiety. The present meta-analytic study was conducted by adopting the Deficit Theory's (Tobias, 1986) claim that a mathematics anxiety emerges as a result of recognition of prior poor mathematics performance or cognition. In the light of the Deficit Theory, the hypothesis of this study an intervention focusing on students' cognitive mathematics anxiety may have potential to reduce their mathematics anxiety was supported by the results of this meta-analytic study. The treatments related to CBT interventions measured in the study were arranged to relieve both expressed worry about the mathematics (cognition element) and emotionally (e.g., fears, tensions) towards mathematics (behavioral element). For example, one of the therapies focusing on both students' cognition and behavioral outcomes in mathematics was implemented by Karimi et al. (2009). The finding of this therapy is located in the present meta-analytic study as a measure of students' mathematics anxiety and its responses to CBT. In this therapy, participants received materials used in sessions at schools and for home practice. The sessions' foci were on identifying what students' negative mathematical thoughts related to their prior poor mathematics performances (three sessions for cognitive mathematics anxiety) and how to cope with negative behaviors associated with negative thoughts in mathematics (six sessions for behavioral part). Like we explained about the relationship between cognitive mathematics anxiety and affective mathematics anxiety in the literature review section, decreasing cognitive mathematics anxiety impacted minimizing students' affective mathematics anxiety (e.g., Karimi et al., 2009; Sharp et al., 2000; Wei, 2010). Therefore, reducing students' mathematical anxiety might be due to the fact that CBT related interventions enable students to become aware of their own negative feelings towards mathematics. This might be the first step for students to overcome their mathematical anxiety. In addition, CBT related interventions implement techniques (e.g., relaxation, desensitization) that may create a growth mathematical mindset rather than a fixed one by reducing their fear, tension, helplessness when they perform in mathematics. After students reduce their mathematical anxiety, they can recognize their own potential to solve mathematical problems and enjoy their mathematics courses. CBT related interventions encourage students to shift their cognitive and affective mathematical feelings from "Mathematics is a subject I am not good" at to "Mathematics is a subject I can perform well." All in all, by considering the Deficit Theory and the findings from the present meta-analytic study, it is possible to conclude that CBT is a helpful intervention on reducing students' cognitive mathematics anxiety and was also helpful to reduce students' affective mathematics anxiety.

\section{IMPLEMENTATIONS}

The findings from this meta-analytic study allowed a more complete picture of how a CBT related environment can help students overcome their mathematics anxiety. These techniques can help teachers implement appropriate CBT related interventions (e.g., relaxation, desensitization) to reinforce their students' mathematical learning by eliminating their existing mathematical anxiety. Not all CBT related interventions are equal, and teachers should implement the most appropriate one based on his/her students' specific mathematics anxiety case. Through implementing the most appropriate techniques of CBT and other interventions have shown a potential to reduce students' mathematical anxiety, teachers can find that a simple change creates a more positive mathematics classroom. The implementation of CBT related interventions to mathematics classrooms provides opportunities for students to become successful not only in mathematics, but also in other subjects as mathematics is considered a gatekeeper for other STEM related fields. When students feel more secure to perform in mathematics, and share their mathematical ideas with others during mathematics instructions, their mathematical learning will be enriched, and they build up confidence, motivation, and preference level in mathematics.

\section{LIMITATIONS}

The present meta-analytic study included studies varying in terms of types of CBT intervention, participants' ages, and duration of intervention. That is one reason a random sampling method was applied to identify any potential moderator analysis. However, the investigation of moderator analysis was not consistent due to the small number of effect sizes in the present study. Further meta-analytic investigations about the effects of CBT on reducing students' mathematics anxiety is required when the number of published studies increases. A note of caution again, the present study presents only 7 studies with 14 effect sizes about the effects of CBT interventions on students' mathematics anxiety; thus, any result of this meta-analysis needs to be considered carefully. 


\section{Disclosure statement}

No potential conflict of interest was reported by the authors.

\section{Notes on contributors}

Ali Bicer - University of Wyoming, USA.

Celal Perihan - Idaho State University, USA.

Yujin Lee - Indiana University-Purdue University, USA.

\section{REFERENCES}

Anderson, V. (2007). An online survey to assess student anxiety and attitude response to six different mathematical problems. In J. Watson and K. Beswick (Eds), Proceedings of the 30th Annual Conference of the Mathematics Education Research Group of Australasia (pp.93-102). Adelaide, SA: Merga.

Ashcraft, M. H. (2002). Math anxiety: Personal, educational, and cognitive consequences. Current Directions in Pychological Science, 11(5), 181-185. https://doi.org/10.1111/1467-8721.00196

Bandura, A. (1994). Self-efficacy. Encyclopedia of Human Behavior, 4(4), 71-81.

Baroody, A. J., \& Costlick, T. T. (1998). Fostering children's mathematical power: An investigative approach to K-8 mathematics instruction. Mahwah, NJ: Lawrence Erlbaum Associates. https://doi.org/10.4324/9781410602084

Barrett, P. M., Dadds, M. R., \& Rapee, R. M. (1996). Family treatment of childhood anxiety: A controlled trial. Journal of Consulting and Clinical Psychology, 64(2), 333-342. https://doi.org/10.1037/0022006X.64.2.333

Bates, A. B., Latham, N., \& Kim, J. A. (2011). Linking preservice teachers' mathematics Self-Efficacy and mathematics teaching efficacy to their mathematical performance. School Science and Mathematics, 111(7), 325-333. https://doi.org/10.1111/j.1949-8594.2011.00095.x

Beck, A. T. (1976). Cognitive therapy and the emotional disorders. New York, NY: International Universities Press.

Beck, A. T., Davis, D. D., \& Freeman, A. (Eds.). (2015). Cognitive therapy of personality disorders. New York, NY: Guilford Publications.

Beilock, S. L., \& Maloney, E. A. (2015). Math anxiety: A factor in math achievement not to be ignored. Policy Insights from the Behavioral and Brain Sciences, 2(1), 4-12. https://doi.org/10.1177/2372732215601438

Beilock, S. L., \& Willingham, D. T. (2014). Math anxiety: Can teachers help students reduce it? Ask the cognitive scientist. American Educator, 38(2), 28-33.

Bernstein, G. A., Layne, A. E., Egan, E. A., \& Tennison, D. M. (2005). School-based interventions for anxious children. Journal of the American Academy of Child \& Adolescent Psychiatry, 44(11), 1118-1127. https://doi.org/10.1097/01.chi.0000177323.40005.a1

Bicer, A., Lee, Y., Capraro, R. M., Capraro, M. M., Barroso, L. R., Bevan, D., \& Vela, K. (2018, October). Cracking the Code: The Effects of Using Microcontrollers to Code on Students' Interest in Computer and Electrical Engineering. In 2018 IEEE Frontiers in Education Conference (FIE) (pp. 1-7). IEEE. https://doi.org/10.1109/FIE.2018.8658617

Birgin, O., Baloğlu, M., Çatlığlu, H., \& Gürbüz, R. (2010). An investigation of mathematics anxiety among sixth through eighth grade students in Turkey. Learning and Individual Differences, 20(6), 654-658. https://doi.org/10.1016/j.lindif.2010.04.006

Cayoun, B. A. (2014). Mindfulness-integrated CBT for well-being and personal growth: Four steps to enhance inner calm, self-confidence and relationships. Hoboken. NJ: John Wiley \& Sons. https://doi.org/10.1002/9781118509111

Cohen, J. (1969). Statistical power analysis for the behavioral sciences. New York, NY: Academic Press.

Cooper, H. (2010). Research synthesis and meta-analysis: A step-by-step approach (4 ${ }^{\text {th }}$ ed.). Thousand Oaks, CA: Sage. 
Deffenbacher, J. L. (1978). Worry, emotionality, and task-generated interference in test anxiety: An empirical test of attentional theory. Journal of Educational Psychology, 70(2), 248-254. https://doi.org/10.1037/0022-0663.70.2.248

Devine, A., Fawcett, K., Szűcs, D., \& Dowker, A. (2012). Gender differences in mathematics anxiety and the relation to mathematics performance while controlling for test anxiety. Behavioral and Brain Functions, 8(33), 1-9. https://doi.org/10.1186/1744-9081-8-33

Downs, S. H., \& Black, N. (1998). The feasibility of creating a checklist for the assessment of the methodological quality both of randomized and non-randomized studies of health care interventions. British Medical Journal, 52, 377-384. https://doi.org/10.1136/jech.52.6.377

Dugas, M. J., \& Robichaud, M. (2007). Cognitive-behavioral treatment for generalized anxiety disorder: from science to practice. New York, NY: Routledge.

Ehrenreich-May, J., Storch, E. A., Queen, A. H., Rodriguez, J. H., Ghilain, C. S., Alessandri, M., ... Wood, J. J. (2014). An open trial of cognitive-behavioral therapy for anxiety disorders in adolescents with autism spectrum disorders. Focus on Autism and Other Developmental Disabilities, 29(3), 145-155. https://oi.org/10.1177/1088357614533381

Erden, M., \& Akgul, S. (2010). Predictive Power of mathematics Anxiety and Perceived Social Support from Teacher for Primary Students' Mathematics Achievement. Journal of Theory and Practice in Education, 6(1), 3-16.

Fennema, E., \& Sherman, J. A. (1976). Fennema-Sherman mathematics attitudes scales: Instruments designed to measure attitudes toward the learning of mathematics by females and males. Journal for research in Mathematics Education, 7(5), 324-326. https://doi.org/10.2307/748467

Foss, D. H., \& Hadfield, O. D. (1993). A successful clinic for the reduction of mathematics anxiety among college students. College Student Journal, 27, 157-157.

Fotoples, R. M. (2000). In my view: Overcoming math anxiety. Kappa Delta Pi Record, 36(4), 149-151. https://doi.org/10.1080/00228958.2000.10518774

Furner, J., \& Berman, B. (2003). Review of research: Math anxiety: Overcoming a major obstacle to the improvement of student math performance. Childhood Education, 79(3), 170-174. https://doi.org/10.1080/00094056.2003.10522220

Hart, L. E. (1989). Describing the affective domain: Saying what we mean. In D. B. McLeod \&V. M. Adams (Eds.), Affect and mathematical problem solving: A new perspective (pp. 37-45). New York, NY: SpringerVerlag. https://doi.org/10.1007/978-1-4612-3614-6_3

Hayes, S. C., Strosahl, K. D., \& Wilson, K. G. (1999). Acceptance and commitment therapy: An experiential approach to behavior change. New York, NY: Guilford.

Hedges, L. V., \& Vevea, J. L. (1998). Fixed- and random-effects models in meta-analysis. Psychological Methods, 3(4), 486-504. https://doi.org/10.1037/1082-989X.3.4.486

Hedges, L. V., Tipton, E., \& Johnson, M. C. (2010). Robust variance estimation in meta-regression with dependent effect size estimates. Research Synthesis Methods, 1(1), 39-65. https://doi.org/10.1002/jrsm.5

Hembree, R. (1990). The nature, effects, and relief of mathematics anxiety. Journal for Research in Mathematics Education, 21, 33-46. https://doi.org/10.2307/749455

Ho, H. Z., Senturk, D., Lam, A. G., Zimmer, J. M., Hong, S., Okamoto, Y., ... Wang, C. P. (2000). The affective and cognitive dimensions of math anxiety: A cross-national study. Journal for Research in Mathematics Education, 362-379. https://doi.org/10.2307/749811

Hoffman, B. (2010). "I think I can, but I'm afraid to try": The role of self-efficacy beliefs and mathematics anxiety in mathematics problem-solving efficiency. Learning and Individual Differences, 20(3), 276283. https://doi.org/10.1016/j.lindif.2010.02.001

Hofmann, S. G., Asnaani, A., Vonk, I. J., Sawyer, A. T., \& Fang, A. (2012). The efficacy of cognitive behavioral therapy: A review of meta-analyses. Cognitive Therapy and Research, 36(5), 427-440. https://doi.org/10.1007/s10608-012-9476-1

House, A. E., House, B. J., \& Campbell, M. B. (1981). Measures of interobserver agreement: Calculation formulas and distribution effects. Journal of Behavioral Assessment, 3(1), 37-57. https://doi.org/10.1007/BF01321350 
James, A. C., James, G., Cowdrey, F. A., Soler, A., \& Choke, A. (2013). Cognitive behavioral therapy for anxiety disorders in children and adolescents. Cochrane Database of Systematic Reviews, 6, 46-90. https://doi.org/10.1002/14651858.CD004690.pub3

Karimi A., \& Venkatesan S. (2009). Cognitive Behavioral Group Therapy in Mathematics Anxiety. Journal of the Indian Academy of Applied Psychology, 35(2), 299-303.

Kendall, P. C. (1993). Cognitive-behavioral therapies with youth: Guiding theory, current status, and emerging developments. Journal of Consulting and Clinical Psychology, 61(2), 235-247. https://doi.org/10.1037/0022-006X.61.2.235

Kendall, P. C., \& Hedtke, K. (2006). Cognitive-behavioral therapy for anxious children: Therapist manual (3rd ed.). Ardmore, PA: Workbook Publishing.

Kendall, P. C., Robin, J. A., Hedtke, K. A., Suveg, C., Flannery-Schroeder, E., \& Gosch, E. (2005). Considering CBT with anxious youth? Think exposures. Cognitive and Behavioral Practice, 12(1), 136-148.Kitchens, A. (1995). Defeating math anxiety. Chicago, IL: Richard E. Irwin, Inc. https://doi.org/10.1016/S10777229(05)80048-3

Lee, Y., Capraro, R. M., \& Bicer, A. (2019). Affective mathematics engagement: A comparison of STEM PBL versus non-STEM PBL instruction. Canadian Journal of Science, Mathematics, and Technology Education, 1-20. https://doi.org/10.1007/s42330-019-00050-0

Lipsey, M. W., \& Wilson, D. B. (2001). Practical meta-analysis. Thousand Oaks: Sage.

Lyons, I. M., \& Beilock, S. L. (2010, November). Mathematics anxiety: Separating the math from the anxiety. Poster presented at the annual Psychonomic meeting in St. Louis, MO. https://doi.org/10.1037/e520592012-606

Ma, X. (1999). A meta-analysis of the relationship between anxiety toward mathematics and achievement in mathematics. Journal for Research in Mathematics Education, 520-540. https://doi.org/10.2307/749772

Ma, X., \& Xu, J. (2004). The causal ordering of mathematics anxiety and mathematics achievement: a longitudinal panel analysis. Journal of Adolescence, 27(2), 165-179. https://doi.org/10.1016/j.adolescence.2003.11.003

Manassis, K., Wilansky-Traynor, P., Farzan, N., Kleiman, V., Parker, K., \& Sanford, M. (2010). The feelings club: randomized controlled evaluation of school-based CBT for anxious or depressive symptoms. Depression and Anxiety, 27(10), 945-952. https://doi.org/10.1002/da.20724

Maskey, M., Lowry, J., Rodgers, J., McConachie, H., \& Parr, J. R. (2014). Reducing specific phobia/fear in young people with autism spectrum disorders (ASDs) through a virtual reality environment intervention. PloS One, 9(7), e100374. https://doi.org/10.1371/journal.pone.0100374

Miller, L. D., \& Mitchell, C. E. (1994). Mathematics anxiety and alternative methods of evaluation. Journal of Instructional Psychology, 21, 353-358.

Moher, D., Liberati, A., Tetzlaff, J., \& Altman, D. G. (2009). Preferred reporting itemsfor systematic reviews and meta-analyses: the PRISMA statement. Annals of Internal Medicine, 151(4), 264-269. https://doi.org/10.7326/0003-4819-151-4-200908180-00135

Namkung, J. M., Peng, P., \& Lin, X. (2019). The relationship between mathematics anxiety and mathematics performance among school-aged students: A Meta-Analysis. Review of Educational Research, 89(3), 459-496. https://doi.org/10.3102/0034654319843494

Neil, A. L., \& Christensen, H. (2009). Efficacy and effectiveness of school-based prevention and early intervention programs for anxiety. Clinical Psychology Review, 29(3), 208-215. https://doi.org/10.1016/j.cpr.2009.01.002

Perihan, C., Burke, M., Bowman-Perrott, L., Gallup, J., Thompson, J., \& Sallese, M. (2019). Effects of cognitive behavioral therapy for reducing anxiety in children with high functioning ASD: A systematic review and meta-analysis. Journal of Autism and Developmental Disorders, 1-15. https://doi.org/10.1007/s10803-019-03949-7

Ramirez, G., Chang, H., Maloney, E. A., Levine, S. C., \& Beilock, S. L. (2016). On the relationship between math anxiety and math achievement in early elementary school: The role of problem solving strategies. Journal of Experimental Child Psychology, 141, 83-100. https://doi.org/10.1016/j.jecp.2015.07.014

Sarason, I. G. (1987). Test anxiety, cognitive interference, and performance. In R. E. Snow \& M. J. Farr (Eds.), Aptitude, learning, and instruction: Volume 3: Cognitive and affective process analyses (pp.131-142). Hillsdale, NJ: Erlbaum. 
Schneider, W. J., \& Nevid, J. S. (1993). Overcoming math anxiety: A comparison of stress inoculation training and systematic desensitization. Journal of college student development, 34(4), 283-288.

Seligman, L. D., \& Ollendick, T. H. (2011). Cognitive-behavioral therapy for anxiety disorders in youth. Child and Adolescent Psychiatric Clinics of North America, 20(2), 217-238. https://doi.org/10.1016/j.chc.2011.01.003

Sharma, S., \& Rao, U. (1983). The effects of self-esteem, test anxiety and intelligence on academic achievement of high school girls. Personality Study \& Group Behavior, 3(2), 48-55.

Steensel, F. J., \& Bögels, S. M. (2015). Cbt for anxiety disorders in children with and without autism spectrum disorders. Journal of Consulting and Clinical Psychology, 83(3), 512-523. https://doi.org/10.1037/a0039108

Tanner-Smith, E. E., \& Tipton, E. (2014). Robust variance estimation with dependent effect sizes: Practical considerations including a software tutorial in Stata and SPSS. Research Synthesis Methods, 5(1), 1330. https://doi.org/10.1002/jrsm.1091

Tobias, S. (1986). Anxiety and cognitive processing of instruction. In R. Wchwarzer (Ed.), Self-related cognitions in anxiety and motivation (pp. 35- 54). Hillsdale, NJ: Erlbaum.

Tobias, S. (1993). Overcoming math anxiety. New York, NY: Norton company.

Uusimaki, L. S., \& Kidman, G. C. (2004, July). Challenging math anxiety: an intervention model. In the 10th International Congress on Mathematical Education (ICME-10), 4-11, Copenhagen, Denmark. https://doi.org/10.11120/msor.2004.04030064

Vitasari, P., Herawan, T., Wahab, M. N. A., Othman, A., \& Sinnadurai, S. K. (2010). Exploring mathematics anxiety among engineering students. Procedia-Social and Behavioral Sciences, 8, 482-489. https://doi.org/10.1016/j.sbspro.2010.12.066

Vukovic, R. K., Kieffer, M. J., Bailey, S. P., \& Harari, R. R. (2013). Mathematics anxiety in young children: Concurrent and longitudinal associations with mathematical performance. Contemporary Educational Psychology, 38(1), 1-10. https://doi.org/10.1016/j.cedpsych.2012.09.001

Wadlington, E., Austin, S., \& Bitner, J. (1992). The treatment of math anxiety and negative math self-concept in college students. College Student Journal, 26(1), 61-65.

Warner, C. M., Warner, C. M., Fisher, P. H., \& Klein, R. G. (2007). School-based behavioral intervention for social anxiety disorder in adolescents: Results of an attention control trial and implementation issues. PsycEXTRA Dataset. https://doi.org/10.1037/e533832007-001

Waxman, H. C., \& Huang, S. Y. L. (1996). Differences by level of technology use on students' motivation, anxiety, and classroom learning environment in mathematics. Journal of Educational Technology Systems, 25(1), 67-77. https://doi.org/10.2190/8HGJ-XRJV-3V9P-KQL5

Wei, Q. (2010). The effects of pedagogical agents on mathematics anxiety and mathematics learning. Doctoral dissertation. Logan, Utah: Utah State University.

West, S., King, V., Carey, T. S., Lohr, K. N., McKoy, N., Sutton, S.F., et al. (2002). Systems to rate the strength of scientific evidence. Evidence Report/Technology Assessment No. 47 (Prepared by the Research Triangle Institute University of North Carolina Evidence-based Practice Center under Contract No. 290-97-001). AHRQ Publication No. 02- E016. Rockville, MD: Agency for Healthcare Research and Quality.

Wigfield, A., \& Meece, J. L. (1988). Math anxiety in elementary and secondary school students. Journal of Educational Psychology, 80(2), 210-216. https://doi.org/10.1037/0022-0663.80.2.210

Wolpe, J. (1961). The systematic desensitization treatment of neuroses. Journal of Nervous and Mental Disease, 132, 189-203. https://doi.org/10.1097/00005053-196103000-00001

Zakaria, E., Zain, N. M., Ahmad, N. A., \& Erlina, A. (2012). Mathematics anxiety and achievement among secondary school students. American Journal of Applied Sciences, 9(11), 1828-1832. https://doi.org/10.3844/ajassp.2012.1828.1832

Zettle, R. D. (2003). Acceptance and commitment therapy (ACT) vs. systematic desensitization in treatment of mathematics anxiety. The Psychological Record, 53(2), 197-215. https://doi.org/10.1007/BF03395440

http://www.iejme.com 